piun und Wilibald Köhler. Darüber hinaus benutzt der Autor auch Texte von Arnolt Bronnen, Franz Jung, Arnold Ulitz sowie Georg Langer. Zum untersuchten Korpus gehört auch das Drama Volk an der Grenze von Rudolf Fitzek sowie einige zehn Gedichte von insgesamt vierzehn Autoren. Man akzeptiert die Erklärung des Autors, dass in einer solchen Arbeit keine Vollständigkeit möglich ist (S. 27). An die Stelle der Vollständigkeit muss aber ein anders Prinzip treten. Der Autor muss erklären, nach welchen Kriterien Romane, Erzählungen, Gedichte und Dramen gewählt wurden. Das tut er nicht. Wenn der Autor betont, dass es sich um ,die Untersuchung eines spezifischen zeitgenösischen Diskursfeldes“ (S. 29) handelt, dann soll hier das Prinzip der Representativität gelten. Es sollten also nicht nur Publikationen mit eindeutig propagandistischer Prägung gewählt werden, sondern auch Werke, in denen Oberschlesien differenzierter dargestellt wird. Unter den analysierten Texten vermisst man vor allem Romane von August Scholtis. Nur ein Fragment aus dem bekanntesten Roman des Schriftstellers wird auf der Seite 234 kurz kommentiert. In der Begründung dieser Marginalisierung des wichtigen Werkes heißt es: „Der „Ostwind“ gehört zwar nicht zum ,Kernkanon“ der Literatur zur oberschlesischen Teilung, jedoch fallen in dem Roman Stellungnahmen auf, die die Entwicklung der sozialpolitischen Lage in Oberschlesien nach dem Ersten Weltkrieg thematisieren.“ (S. 234) Wie lauten die Kriterien des „Kernkanons“, welche Autoritäten bestimmen den Kanon, aus dem Romane von Scholtis ausgeschlossen wurden? Ein solcher Kanon ist ohne Scholtis undenkbar und die überschaubare Sekundärliteratur zu Scholtis würde das Bild des analysierten Gegenstands deutlich modifizieren. Das Buch von Janikowski ist ein gescheiterter Versuch, den geisteswissenschaftlichen Diskurs über Oberschlesien zu vertiefen, denn die forcierte Psychologisierung der Literatur und Publizistik verstellt dem Autor den Blick auf literaturhistorische Zusammenhänge und beeinträchtigt die analytische Präzision.

Robert Rduch

\title{
Marcus Stiglegger: Auschwitz-TV Reflexionen des Holocaust in Fernsehse- rien. Springer VS: Wiesbaden: Springer 2015, 95 S.
}

DOI: $10.19195 / 0435-5865.141 .36$

Marcus Stiglegger zeigt in seinem Band Auschwitz-TV Reflexionen des Holocaust in Fernsehserien einen geschichtlichen Abriss der Auschwitz-Darstellungen in dem Medium - Fernsehserie. Der Analyse werden die filmischen Inszenierungen der Grausamkeit, der Täter, der Opfer und ihrer Gefühle unterzogen. Das Ziel dieser Darstellungen ist nach Meinung des Verfassers durch den Einsatz bestimmter Bilder auch bestimmte Emotionen bei dem sich regelmäßig vor den Fernsehapparaten versammelten Publikum hervorzurufen. In den einschlägigen gegenwärtigen Publikationen wird dabei auf eine bedeutende Gefahrenquelle hingewiesen: Die mediale Emotionalisierung von Gewaltszenen kann auf den Zuschauer unfreiwillig euphorisierend wirken. ${ }^{1}$ Stiglegger sucht nach Ant-

${ }^{1}$ Vgl. Marcus Stiglegger: Ritual und Verführung. Schaulust, Spektakel und Sinnlichkeit im Film. Berlin 2006; Carsten Gansel / Heinrich Kaulen (Hrsg.): Kriegsdiskurse in Literatur und Me- 
worten auf die Fragen, wie in den Medien kriegerische Gewalt dargestellt wird, wie sie ästhetisiert oder auch moralisch wie politisch zu bewerten ist. Auch aus den früheren Arbeiten von Stiglegger wird die Erkenntnis gewonnen, dass Gewalt nicht weit von Lust, auch von der erotisierenden Lust anzusiedeln ist. ${ }^{2}$ Deswegen sind Filme, auch die, die das Thema Auschwitz betreffen, immer mehrdeutig in unterschiedlichen geschichtlichen Zeiten und kulturellen Räumen zu deuten.

In seinem Band Auschwitz-TV Reflexionen des Holocaust in Fernsehserien untersucht Stiglegger den Einfluss narrativer Fernsehserien auf die Erinnerungskultur im deutschsprachigen Kulturkontext. Dabei geht es dem Autor nicht in erster Linie um die deutschen Produktionen, sondern er wirft den Blick auch auf internationale Fernsehserien. Den Ausgangspunkt seiner Forschungen bildet das Jahr 1978 - jenes, so die Festsetzung des Autors, „Schlüsseljahr“ (S, VII) für die Entwicklung der medialen Auseinandersetzung mir dem Thema Auschwitz -, in dem die US-Serie Holocaust ausgestrahlt wurde. Sie wurde alleine in den USA von 100 Millionen Zuschauer verfolgt und in West-Deutschland erreichte sie 1979 ein Publikum von 16 Millionen. Stiglegger zeigt an diesem Stoff auf, wie durch das in der Serie erzeugte Bild der historischen Ereignisse, Inszenierungen der Auschwitz-Thematik in weiteren Serien dieses Genres beeinflusst wurden.

Die analytische Basis Stigleggers Forschungen bildet der von ihm entwickelte Ansatz einer Seduktionstheorie des Films, der darin besteht, die komplexen Manipulations- und Vereinnahmungsstrategien des Mediums zu untersuchen. ${ }^{3}$ Den Untersuchungsstoff bilden vor allem Serien, die sich explizit „,der aus dem populären Bildarchiv geschöpften Ikonographie des Holocausts bedienen: Standardsituationen wie Verhaftung, Ghettoleben, Depotration, Lageralltag, Vernichtung und Tortur, sowie Flucht“" (S, VII). Die Analyse wird an folgenden Serien durchgeführt: Am grünen Strand der Spree (D 1960, WDR), Das Haus in der Karpfengasse (D 1963, WDR/Independent-Film GmbH), Stawka wieksza niż życie/ Sekunden entscheiden (P1 1967/68, Telewizja Polska TVP/ Zespół Realizatorów Filmowych Syrena), Holocaust/ Holocaust - Die Geschichte der Familie Weiß (USA 1978, NBC), Archiv des Todes (DDR 1982, MDR), Ein Stück Himmel (D 1982, WDR), Heimat (D 1982 ff., Edgar Reitz Film/SFB/WDR), Au Nom de Tous Miens/Martin Grey - Schrei nach Leben (F/K 1985, Les Productions Mutuelles Ltée, Producteurs Associés, TF1 Films), War and Remembrance/ Feuersturm und Asche (1988, Dan Curtis Productions/ABC Circle Films/Jadran Film), Klemperer - Ein Leben in Deutschland (D 1999, MDR/ Studio Babelsberg), Madar-e sefr daradscheh (Iran 2006/2007), Band of Brothers/ Band of Brothers - Wir waren wie Brüder (USA 2009, HBO), Unsere Mütter, unsere Väter (D 2013, ZDF/ team Worx). Vor dem Hintergrund dieses deutschen, amerikanischen, französischen, polnischen und iranischen Filmmaterials untersucht Stiglegger zum einen die Thematisierung und zum anderen die Inszenierung des Holocaust. Den ersten Schwerpunkt des Bandes bildet die Dokumentation der historischen Entwicklung vom Fotodokument über die frühe kinematographische Holocaust-Ikonografie bis hin zur Popularisierung dieser Bildwelt.

dien von 1989 bis zum Beginn des 21. Jahrhunderts. Göttingen 2011; Jan Süselbeck: Im Angesicht der Grausamkeit. Emotionale Effekte literarischer und audiovisueller Kriegsdarstellungen vom 19. bis zum 20. Jahrhundert. Göttingen 2013.

${ }^{2}$ Vgl. Marcus Stiglegger: Sadiconazist. Sexualität und Faschismus im Film. 2. Auflage St. Augustin 2002 (1999); Stiglegger, Ritual und Verführung, 2006.

${ }^{3}$ Vgl. Stiglegger, Ritual und Verführung, 2006. 
Daraus tritt eine erste These für den Band hervor, die besagt: Seit 1945 besteht ein permanentes Wechselverhältnis zwischen dokumentarischen, kinematographischen und fiktionalen TV-Aufarbeitungen der Holocaust-Thematik, die 1978 in der Serie Holocaust kulminiert und danach wiederum in alle drei Formate (Dokumentation, Kinofilm, TV-Serie) hineinwirkt. So sieht der Autor in den später entstandenen Inszenierungen eine Tendenz der Bezugnahme eher auf die bereits entworfenen Bilder, audiovisuellen Verdichtungen früherer Fiktionalisierungen als auf die historischen Bilddokumente. Der Autor kommt zu der Auffassung, dass die fiktionalisierten Geschichtserzählungen den Diskurs der Historiker teilweise überlagern und nicht nur die ikonischen Bilder durchsetzen sondern auch sich für eine Etablierung neuer Terminologie verantwortlich machen lassen. Die letzte Behauptung wird an dem Terminus „Holocaust“ überzeugend bewiesen. Unter Rückgriff auf die Arbeiten Roland Barthes sieht Stiglegger in dem so entstandenen, durch Medien geprägten und gespeicherten kollektiven Bildgedächtnis eine Form vom „modernen Mythos“ über die Geschichte.

Aus der Untersuchung und Vergleichsanalyse der internationalen Fernsehserien wird eine weitere These hergeleitet: Die Fiktionalisierungen des Holocaust werden in den nationalen Diskursen unterschiedlich vorgenommen. Die etablierten Bilder werden in neuen Kontexten eingesetzt und damit von ihrer ursprünglichen Semantik im weiten Ausmaße befreit. Als Beispiel führt Stiglegger die DDR-Serie Archiv des Todes, in der die Kriegsverbrechen der Nazis deutlich dargestellt werden, die spezifische Judenverfolgung aber nicht angesprochen wird. Die jüdischen Opfer finden keinen Eingang in diese Inszenierung, dafür wird um so mehr Platz den ,,antifaschistischen Häftlingen“ eingeräumt. An diesem Manöver lässt der Autor den Einfluss der SED-Politik vermuten. Stiglegger arbeitet des weiteren auch die spezifischen nationalen Unterschiede zwischen den deutschen und den amerikanischen Fernsehserien heraus. Für den polnischen Leser wird sicherlich von Interesse sein, die Ausführungen Stigleggers zu der polnischen Serie Stawka większa niż życie zu verfolgen. Zum einen wird für die Serie die ambivalente Faszination für den deutschen Feind diagnostiziert, an anderer Stelle nennt Stiglegger diese Darstellungsform auch Nazi Chic ${ }^{4}$, zum anderen wird die fehlende Auseinandersetzung mit dem Thema Holocaust herausgestellt, dafür bringt der Autor einen folgenden Grund an: ,[...] in Polen dürfte das Interesse an einer Auseinandersetzung mit dem Holocaust selbst begrenzt sein, denn immerhin wurden in diesem Land die Todeslager Auschwitz und Birkenau mit Hilfe einheimischer Kräfte betrieben“ (S. 79). Die Rezensentin würde an dieser Stelle andere Argumente vorziehen, die auch in der Forschung Belegung finden würden.

Die Publikation besteht aus sieben Kapiteln, die drei ersten sind der Begriffsbestimmung und der Einführung in das Thema gewidmet, vier weitere beschäftigen sich mit den nationalen Differenzierungen in der Darstellung des Holocausts. Zu Beginn in Fernsehserien als populäres Bildarchiv (1-8) eröffnet Stiglegger eine Diskussion der Begrifflichkeit und der Bedeutung von Film und Fernsehserien für das kollektive Gedächtnis. Dazu konstatiert der Autor: „Medien dienen der Speicherung, aber auch der Überlieferung vom Wissen aus der Vergangenheit und über die Vergangenheit. Die mediale Form und die Rahmenbedingungen der Medienproduktion prägen die gespeicherten und vermittelten Inhalte - und somit auch das Wissen über die Vergangenheit“" (S, 5) Im Kapitel 2. unter

${ }^{4}$ Stiglegger, Marcus: Nazi Chic \& Nazi Trash. Faschistische Ästhetik in der Populärkultur. Berlin 2011. 
dem Titel Entstehung einer audiovisuellen Holocaust-Ikonografie (9-27) folgt ein filmhistorischer Exkurs, der die Holocaust-Ikonografie aus filmischen Darstellungskonventionen herleitet und die wechselseitige Beeinflussung zwischen Fernsehen und Film nach der Ausstrahlung der Serie Holocaust (1978) herausstellt. Daran schließt sich eine Abhandlung an Mediale Bilder als historische Simulakren (29-34), in der die Theorien von Jean Baudrillard und Roland Barthes auf das Medium Serie angewendet werden. In diesem Abschnitt werden die reinszenierten historischen Szenarien als historische Simulakren definiert. Im Kapitel 4, Die TV-Serie „Holocaust“. Die Geburt eines medialen Diskurses (35-46) nimmt der Verfasser einen direkten Bezug auf die amerikanische Holocaust-Serie, wo er viel Platz der Analyse der spezifischen Holocaust-Ikonografie widmet wie auch die deutsche und internationale Rezeption der Serie diskutiert. Im weiteren Kapitel Der Holocaust in deutschen Fernsehen. Alltagsbilder aus dem Nazireich (47-64) ist der Verfasser um eine vergleichende Analyse deutscher Fernsehserien mit dem amerikanischen Erfolgsrezept bemüht. Hier werden die ikonographischen Ähnlichkeiten und auch grundsätzliche konzeptuelle Unterschiede im Umgang mit dem Thema herausgestellt. Kapitel 6, Der Holocaust im amerikanischen Fernsehen. Vom Hyperrealismus zum historischen Simulakrum (65-76) liefert den Abriss der Entwicklung der amerikanischen Serienproduktion nach der Holocaust-Serie. Im nachfolgenden und letzten Kapitel Präsenz des Holocaust im internationalen Fernsehen (77-87) wird das Augenmerk auf die internationalen Produktionen gelenkt. Hierbei werden Fernsehserien aus Frankreich, Polen und der DDR ausgewertet. Dabei stellt der Verfasser fest, dass sich ,in der sozialistischen Nachkriegszeit eine teilweise andere Einschätzung der historischen Fakten etabliert hatte" (S, IX).

Der von Marcus Stiglegger vorgelegte Band liefert einen umfassenden Einblick in die Reflexion zum Thema Holocaust und Auschwitz-Darstellungen in deutschen und internationalen Fernsehserien. Wo gelebte Erfahrung sukzessive verschwindet, gewinnen fiktionale Formen eine neue Bedeutung, und es beginnt eine neue Generation zu sprechen. Stiglegger geht nach Jan Assmann von der Prämisse aus, dass die Zeugen als Träger des kommunikativen Gedächtnisses fungieren. Den Ansatz aufnehmend, gilt es für den Autor angesichts der vorliegenden Thematik nicht nur den Übergang vom kommunikativen zum kulturellen Gedächtnis zu untersuchen, sondern umgekehrt auch jene Prozesse in den Blick zu nehmen, in denen Bestandteile des kulturellen Gedächtnisses auf das kommunikative Gedächtnis einwirken. Kurz gefasst, Stiglegger zeigt am Beispiel der Fernsehserien, dass die Vergangenheit des Holocausts in dieser Form existiert, wie sich Menschen an sie erinnern. Der soziale Rahmen und eine spezifische Wissensordnung prägen und determinieren den Prozess des Bewusstwerdens der Vergangenheit. Innerhalb einer Gemeinschaft, der amerikanischen, der deutschen oder polnischen wird so ein kohärentes Wissen mit räumlichem und zeitlichem Gültigkeitsanspruch konstruiert. Dieses Wissen umfasst die Kenntnisse über die Vergangenheit und dient etwa als Basis für die Konstruktion einer kulturellen Identität. (Vgl. S, 5)

Monika Wolting

Germanica Wratislaviensia 140, 2016

(C) for this edition by CNS 